\title{
Design of spectrometer based on the Internet of things
}

\author{
Longyang Zheng ${ }^{1, a}$, Jie Sun ${ }^{2, b}$, Haiqiang $\mathrm{Wu}^{3, \mathrm{c}}$
}

The key laboratory of film electronic and communication device, Electronic and information

engineering college, Tianjin University of Technology, Tianjin300384, China

a918593814@qq.com, bsun_jie_mail@126.com, ${ }^{\mathrm{a}} 963646625 @ q q . c o m$

\section{Keywords: Architecture of the internet of things, Spectrometer, RFID, Remote control}

Abstract:The features, architecture, hardware connection, application software and the transfer protocol about the spectrometer based on the internet of things are presented in this paper. Applying the technology of the internet of things, it can enhance the real-time and remote control ability of spectrometer, improve the production efficiency and quality. This spectrometer based on the internet of things will play a more important role in the industrial production.

\section{Introduction}

Internet of things is considered to be the third wave of the information industry after the computer, Internet and the mobile communication network. Along with the trend of the IT innovation, the Internet of things has broad application including intelligent transportation, environmental protection, government service and public security. It makes important contributions to the allocation of resources, economy development and industrial upgrading.[1,2]

Spectrometer has high sensitivity, which becomes the best method to detect the high-molecular compound and biological substance, and also plays an important role in many fields.[3] Traditional spectrometer belongs to precise laboratory instrument, it is difficult to work in the harsh environment, can not realize on-line measurement and can not share the data collected in time. Spectrometer based on the Internet of things can effectively solve the difficulties encountered in industrial application and extend the spectrum measurement function.

\section{The character of spectrometer based on the Internet of things}

Besides the traditional functions, the spectrometer based on the Internet of things can also get information of the material tested by the RFID and make the managers and decision makers to obtain spectral data throng the network no matter when and where they are. Comparing with the traditional spectrometer, the spectrometer based on the Internet of things has the following special features.

Remote control.

The spectrum data collected can be transmitted to remote client for observation and analysis through the internet according to the transfer protocol; the control commands from the remote client can also been sent to the spectrometer which parses the commands according to the transfer protocol and reacts in terms of commands such as setting the parameters of spectrometer or doing measurements. [4] With the remote control function, the spectrometer can work easier in the toxic or polluted occasions, and make the tester has no limit on the geographical position, and increase the flexibility of the spectral measurement.

On-line monitoring the specific subject.

The traditional spectrometer can only obtain the spectral data, can not record the source and the serial number of the material. Under the Internet of things technology, RFID is integrated in the 
spectrometer to get the description of the material being tested.[5] It is very favorable for on-line monitoring, for example, in the pharmaceutical production process, it is easy to record serial number of unqualified product and discard it.

Multiple information fusion measurement.

Sometimes the spectrometer is needed to analyze various properties of the material at the same time, off-line detection usually has a lag, can not accurately describe the real-time process and the tedious separation process usually produces error in results. Internet of things technology can make the multiple spectrometers under the different spectral band as a measurement system, and join together of the spectral data which each spectrometer acquires. Then all the data are send to server, the server analyzes the data by use of the designed model and gets the results which reflect the material information. for instance, in the pharmaceutical production process, every step requires measuring material physical characterization including solid grain size, humidity, mixing uniformity, viscosity, hardness and so on. Off-line measurement which is tested one term by one term by the traditional spectrometer can not present the character of different properties at the same time, while the spectrometer under the internet of things can finish the real-time and fusion measurement.[6,7]

The whole process quality control.

That the spectrometer based on the internet of things analyses material composition in every step of industrial production can effectively monitor the product quality. For example, there are a series of operations including the medicine mixing, chemical reaction, forming and packing in the process of the pharmaceutical preparation and one mistake occurring in any step may have serious consequence. Using Internet of things spectrometer with on-line product quality inspection and production scheduling system, intelligent decision system and alarm system can timely access the material condition, the material properties and the process information, make optimum decision for the material consumption, energy consumption in the whole process of the production.

\section{The architecture of spectrometer based on the Internet of things}

The architecture of Internet of things includes the perception layer, transmission layer, processing layer and the application layer as shown in Figure 1. [8] The architecture of spectrometer based on Internet of things is similarly designed.

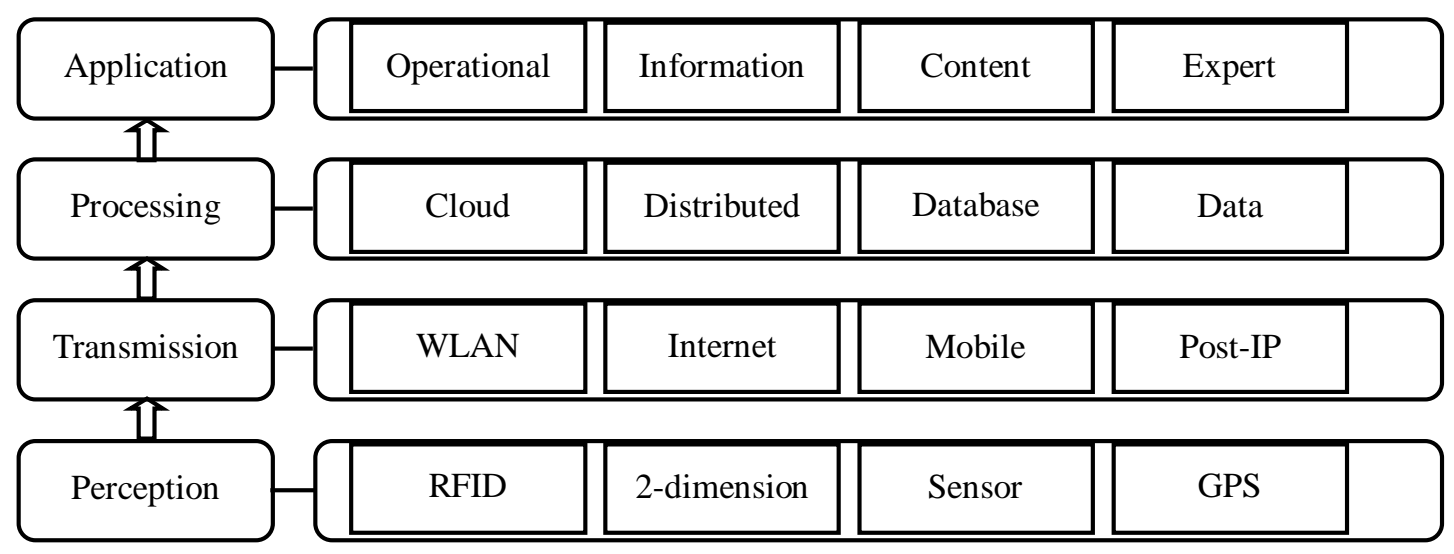

Figure 1 The architecture of the Internet of things

The data perception layer

Data perception layer's function is used to sense the environment around the device and own 
state, process and judge the perception information, respond according to the corresponding rules, and send the intermediate results or the final result to the transmission layer. The perception layer is in the front of the Internet of things, in addition to capturing the real world information, also can control the object processed. Therefore, it is also known as the interactive perception layer .[9] The spectrometer's function in the perception layer mainly includes the spectral data acquisition, the record of measured material information, spectral measurement parameter setting.

The spectral data acquisition is completed by the photoelectric conversion module and the A/D conversion module. the RFID is used to record the static attributes, such as the serial number, quality, color, etc. Before the spectrometer runs, there are some parameters needed to be set such as scanning type, scanning rate, scanning step, slit width, amplification factor and so on.

Before the data collected are transferred to the transmission layer, the encapsulation of the data must be done according to the standardized format. One frame of the spectrum data transmitted in the network should include the initial domain, the measured material property domain, the control type or data type domain, the data domain and the check code. The initial domain is the character description of network spectrometer, the attribute domain records static properties of the measured material, such as the serial number, state and so on. The frame length describes the frame size. Due to the spectrometer's operation is different, the length of the data description is also different, so the frame length is not fixed. The control type indicates the operations to spectrometer. The classification of the control types include the motor control, analog switch control, A/D control, PMT voltage control. Data domain is not fix length, which is beneficial to improve the flexibility and scalability of the protocol. Check code plays an indispensable role in guaranteeing the accuracy of the transferred information.

The data transmission layer

The data transmission layer is responsible for the transfer of the data between the perception layer and the processing layer. It provides an information channel to realize the purpose that all the devices can be connected.

The transfer method of spectrometer based on the internet of things can be divided into cable access and wireless access according to the different working environment. If the spectrometer takes the cable access, an network chip can be embedded in the spectrometer, which is used to make the MAC address identification, data frame assembly/split and transceiver, CRC code calibration, timeout retransmission, link integrity test, etc. If the spectrometer takes the wireless access, WIFI module or ZIGBEE module will be integrated in the spectrometer.

The data processing layer

The data processing layer provides the public data storage and processing functions for the application system of the Internet of things. Under the support of the high performance computing technology, the vast amounts of information is processed in real-time, also the data mining, store, management, control is done. It provides an efficient and reliable technology support platform for the upper management and industry application. The processing layer of spectrometer under the internet of things mainly completes the functions of data storage and data mining.

The data storage is some tables created for the collected data, such as spectral data table, parameters setting of the spectrometer table, etc. In the table, at least one valid keyword is needed for convenient search, and the index is needed to improve the analysis speed. The data mining is that processing layer gets the useful, potential, unknown information from a large amount of data. There are two aspects about data mining in the spectrometer based on internet of things. One is that the processing layer analyzes the tested material depend on the spectrum, the other is that it finds the characteristic and law from the spectral data to guide the production. 
The application layer

The management platform and running platform is constructed in the application layer. The operating interface is different as the different industry asks to the spectrometer, but several parts must be included such as the client login, equipment register, spectrum display, spectrometer control, etc. The spectrometer application layer under the Internet of things is a deep integration of network and the professional spectrometer technique. In addition, in order to provide accurate information service, the professional knowledge of the spectrometer must combine with the industry production management, information management, organization and scheduling.

\section{The implementation of spectrometer based on the Internet of things}

The hardware structure. The structure of spectrometer based on the Internet of things should include spectral acquisition module, motor control module, analog signal processing module, RFID module and network transmission module. The first three are the functions of the traditional spectrometer, network transmission module and RFID module are the hardware characters of the spectrometer under the Internet of things, so only the two parts are introduced. ARM11 is adopted as the main controller chip of the spectrometer and network access is the cable connection.

The network transmission module

The network transmission module is composed by the network control chip DM9000, transformer H1101, and network interface RJ-45. The specific connection is shown in Figure 2. Network control chip realizes the functions of Ethernet access and the physical medium access, including MAC address recognition, assembly/split and send or receive data frames, timeout retransmission, signal polarity detection and link integrity test, etc.[10] Network transformer is mainly used to enhance signal, make further transmission distance, isolate chip from external. Network interface realizes connection with external network.

Spectrometer main controller Network control chip Network transformer Network interface

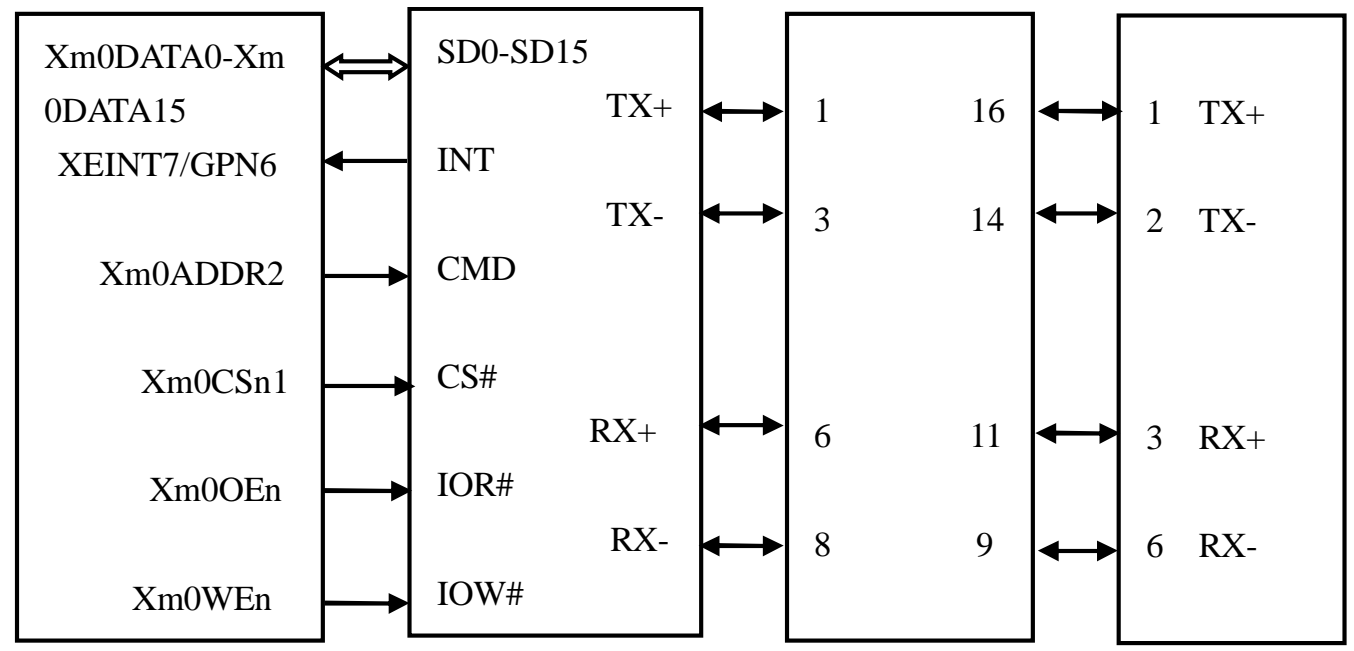

Figure 2 Connection of network hardware

The radio frequency identification(RFID) module

The radio frequency identification module includes reader-writer and electronic tag, the connection is shown in Figure 3. The working steps are that, firstly, reader-writer is power on and then emits electromagnetic wave in certain area. Secondly, when the electronic tag goes into the 
area, it receives the reader-writer signals, and the data analyzed by the demodulation circuit from signals are sent to the data logic control part in where complete data storage and other operations. Thirdly, reader-writer receives the data that electronic tag sends and checks the data validity by decoding and error checking .[11] Lastly, reader-writer sends data which have been verified to the spectrometer main controller. It is worth mentioning that there is a level switch chip needed to convert electronic level between the main controller and the reader-writer.

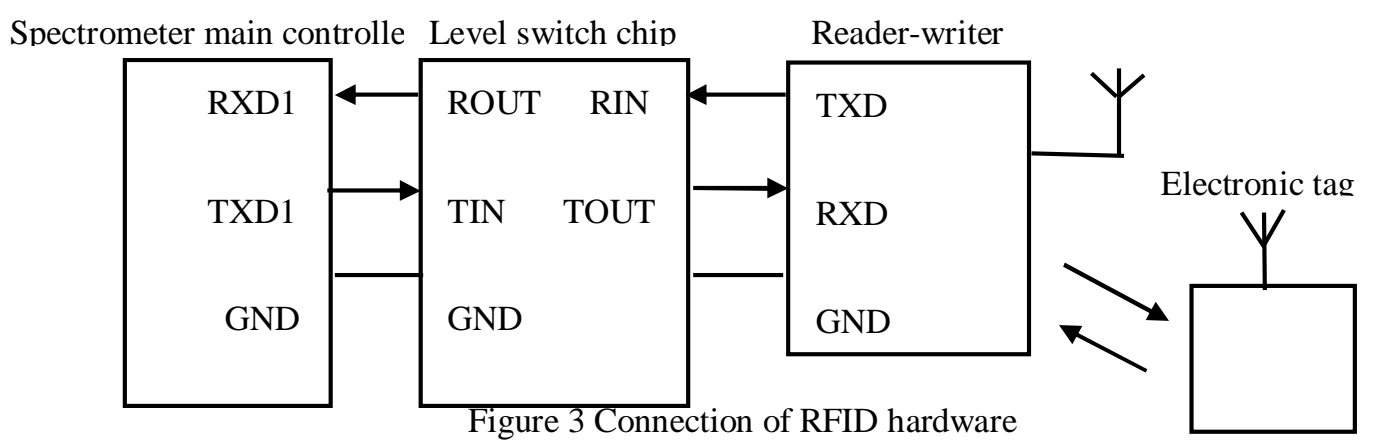

\section{The application software}

The communication protocol

The communication protocol is the standard of the data format for remote communication between the client and the spectrometer. Only when both sides follow the provision of the data exchange can network ensure communication smoothly. The communication protocol complies with the principles of reliability and scalability, and refers to the standard of Ethernet protocol. Table 1 and table 2 is the definition of data frame between the client and the spectrometer.

Table 1 The definition of the downward transfer data frame

\begin{tabular}{|c|c|c|c|c|}
\hline Domain name & \multicolumn{2}{|l|}{ Code } & Data type & Size \\
\hline Initial domain & \multicolumn{2}{|l|}{$0 \mathrm{X} 42$} & HEX & 1 byte \\
\hline Length domain & \multicolumn{2}{|l|}{ Frame length } & HEX & 1 byte \\
\hline \multirow{3}{*}{$\begin{array}{l}\text { Attribution } \\
\text { domain }\end{array}$} & & & 1 byte \\
\hline & \multicolumn{2}{|c|}{$\begin{array}{l}\text { Measured objectnumber } \\
\text { Measured object state }\end{array}$} & HEX & 1 byte \\
\hline & \multicolumn{2}{|c|}{ Motor control } & & \\
\hline \multirow{6}{*}{$\begin{array}{l}\text { Control } \\
\text { domain }\end{array}$} & \multicolumn{2}{|c|}{ Analog switch control } & $\mathrm{BCD}$ & 1 byte \\
\hline & \multicolumn{2}{|c|}{ A/D control } & $D C D$ & Toyle \\
\hline & \multicolumn{2}{|c|}{ PMT voltage control } & & \\
\hline & & Number & $\mathrm{BCD}$ & 1 byte \\
\hline & Motor control & Steps & & 2 bytes \\
\hline & type & Speed & HEX & 2 bytes \\
\hline \multirow[t]{4}{*}{ Data domain } & & Control function & & 1 byte \\
\hline & \multicolumn{2}{|c|}{ Analog switch magnification } & HEX & 2 bytes \\
\hline & \multicolumn{2}{|c|}{ A/D control } & HEX & 1 byte \\
\hline & \multicolumn{2}{|c|}{ PMT voltage control } & HEX & 1 byte \\
\hline Check code & \multicolumn{2}{|l|}{$\mathrm{CS}$} & HEX & 1 byte \\
\hline End domain & \multicolumn{2}{|l|}{$0 \times 24$} & HEX & 1 byte \\
\hline
\end{tabular}


Table 2 The definition of the upward transfer data frame

\begin{tabular}{|c|c|c|c|c|}
\hline Domain name & \multicolumn{2}{|l|}{ Code } & Data type & Size \\
\hline Initial domain & \multicolumn{2}{|l|}{$0 \times 42$} & HEX & 1 byte \\
\hline Length domain & \multicolumn{2}{|l|}{ Frame length } & HEX & 1 byte \\
\hline Attribution & \multicolumn{2}{|c|}{ Measured object number } & HFY & 1 byte \\
\hline domain & \multicolumn{2}{|c|}{ Measured object state } & HLA & 1 byte \\
\hline \multirow{5}{*}{$\begin{array}{l}\text { Data type } \\
\text { domain }\end{array}$} & \multicolumn{2}{|l|}{ Operation state } & & \\
\hline & \multicolumn{2}{|l|}{ Motorlocation } & $R C D$ & 1 hute \\
\hline & \multicolumn{2}{|c|}{$\mathrm{A} / \mathrm{D}$ acquisition data } & $D C D$ & 10yle \\
\hline & \multicolumn{3}{|l|}{ PMT voltage } & \\
\hline & Operation state & & & 1 byte \\
\hline \multirow[t]{3}{*}{ Data domain } & Motorlocation & $\begin{array}{l}\text { Number } \\
\text { Location }\end{array}$ & HEX & 3 bytes \\
\hline & \multicolumn{2}{|c|}{$\mathrm{A} / \mathrm{D}$ acquisition data } & & 2 bytes \\
\hline & \multicolumn{2}{|l|}{ PMT voltage } & & 1 byte \\
\hline Check code & \multicolumn{2}{|l|}{$\mathrm{CS}$} & HEX & 1 byte \\
\hline End domain & \multicolumn{2}{|l|}{$0 \times 24$} & HEX & 1 byte \\
\hline
\end{tabular}

The flow chart of application program

Spectrometer receives the commands from the client and parses them depend on the communication protocol and makes the different responses according to the request from client, the flow chart is shown in figure 4.

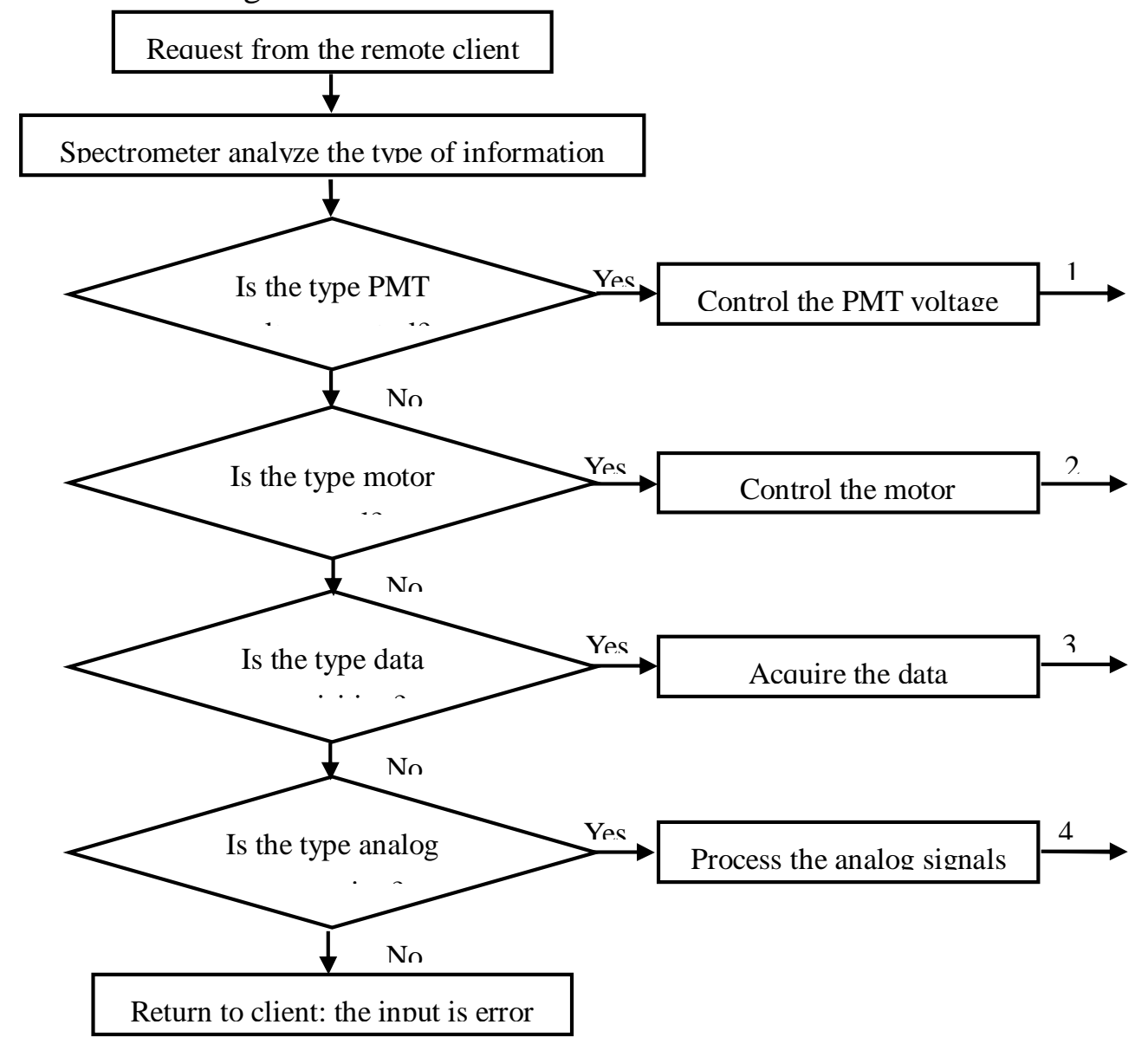

Figure 4 The flow chart of function analysis 
Follow the flow chart, the spectrometer can complete the control from the client and feedback information. The detailed flow chart can not be presented because of the limit of space.

\section{Conclusion}

This paper describes the design of spectrometer based on the internet of things including the hardware connection and software structure. The Internet of things has abilities to perceive all of objects and transfer information reliably and intelligently control, makes it possible to get the real-time information of object, so the application rang of spectrometer under the technology of internet of things must be greatly enlarged.

\section{Reference}

[1]Draft C, October, Kranenburg R V, et al. The internet of things[J]. IEEE Eighth International Conference on Intelligent Sensors, Sensor Networks \& Information Processing, vol.46, no.4, pp.333-338, 2013.

[2]Hongbo zhu,et al. Survey on the internet of things[J]. Journal of Nanjing University of Posts and Telecommunications( Natural Science), vol.31, no.1, pp.1-9, 2011.

[3]Anbing zhao. Design of measurement and control system with ARM for fluorescence spectrophotometer[D].Tianjin:Tianjin university of technology, 2013.

[4] Zheng L. Design and realization of online real-time monitoring system for swing-ARM of infrared spectrometer[J]. Computer Applications and Software, vol.29, no.1, pp.220-223, 2012.

[5]Changpeng He. RFID application research for discrete manufacturing[J]. Jisuanji Jicheng Zhizao Xitong, vol.20, no.5, pp.1160-1170, 2014.

[6]Xiaoliang Wang, et al. Applications of near infrared spectrometer in the pharmacy[J]. Northwest Pharmaceutical Journal, vol.24, no.3, pp.228-230, 2009.

[7]Guoliang Li, et al. Applications of Raman Spectrometer in Process Monitoring[J], Guangdong Chemical Industry, vol.35, no.5, pp.106-109, 2008.

[8]Gubbi J, Buyya R, Marusic S, et al. Internet of Things (IoT): A vision, architectural elements, and future directions[J]. Future Generation Computer Systems, vol.29, no.7, pp.1645-1660, 2013.

[9]Yigang Han, et al. The internet of things[M]. China machine press, 2012.

[10]Jiaqi Sun,et al. Network principle and application[M].Beijing institute of technology press, 2010.

[11]Lei Wang. Research the application of RFID chips in internet of things[J]. Netinfo security, no.5, pp.64-67, 2012. 